\title{
Segmentation of Suspicious Region using GAN Based CNN in Brain MR Images
}

\author{
Preethi Nanjundan, W. JaiSingh
}

Abstract: Generative Adversarial networks (GANs) are algorithmic architectures that use dual neural networks, pitting one in obstruction to the other (therefore the "opposing") with a intent to produce new, artificial times of evidences that can avoid for real proofs. They are used significantly in image group. In the scope of therapeutic imaging, creating precise technical impulsive shots which are dissimilar from the Adversarial exact ones, signify an inspiring and esteemed goal. The consequential artificial pics are probably to expand analytical reliability, permitting for data augmentation in computer-aided estimation in addition to medic trial. There are optimistic hard states in producing unreal multi-collection awareness Magnetic Resonance (MR) photos. The main trouble being low difference MR photos, dynamic steadiness in attention framework, and private-series volatility. In this paper, we realization on Generative Networks (GANs) for generating artificial multi-series attention Magnetic Resonance (MR) images. This comprises snags largely as a result of small dissimilarity MR pictures, durable correctness in Brain composition, and private-series inconsistency. This effort proposes a kind novel GAN founded deep learning mark that syndicates GAN group, augmentation, detection and gathering of suspicious regions. The proposed stroke is measured with the aid of pictures developed from BRATS (Multimodal Brain Tumour Image Segmentation Challenge) and dataset IXI in 2015. The usefulness of the future process is added and the outcomes are discussed limited the paper.

Keywords : Generative Adversarial Networks, Artificial Health Picture Group, Brain MRI, Facts Augmentation, Surgeon Tool, Graphic Turing Trial.

\section{INTRODUCTION}

There are positive difficult conditions in generating artificial many-sequence Brain Magnetic Resonance (MR) picture. The primary difficulty being small difference MR pictures, durable reliability in Brain framework, and private-collection inconsistency. The versatility of GAN in producing odd pictures makes it well-known for many picture processing tasks. In addition with predictable approaches [1], Convolutional Neural Networks (CNNs) take these times reformed health picture investigation [2], such as Brain Magnetic Resonance Imaging (MRI) subdivision [3]. Nevertheless, CNN instruction weights large health facts

Revised Manuscript Received on April 25, 2020.

* Correspondence Author

Preethi Nanjundan1*, Department of Computer Science, Christ(Deemed to be University), Lavasa, Pune, India.

W. JaiSingh, Department of Computer Applications, Kumaraguru College of Technology, Coimbatore, Tamilnadu, India

(c) The Authors. Published by Blue Eyes Intelligence Engineering and Sciences Publication (BEIESP). This is an open access article under the CC BY-NC-ND license (http://creativecommons.org/licenses/by-nc-nd/4.0/) which can be painstaking and time intense to gain [5]. Towards impressed this subject, facts augmentation systems with the support of the method of reconstructing original pics are not infrequent for complex performance, containing of geometry and strength transformations [6, 7].The main task near this purpose is that the same old GAN prototypical was to jump with planned for creating impulsive shots from arbitrary clatters, henceforth its incapable to yield actual pictures for slightly further-handle. A not uncommon exercise is to reverse a specified portrait over to a latent code such that it is bright to be reconstructed by the generator. Thus, permitting the reversed encryption for practice for extra dispensation. To opposite the group progression, there are dual current strategies. Distinct is at when adjust the hidden encryption by means of lessening the reform fault done returned-extension $[17,12,4]$. The additional is to instruct a additional convert to grounding the recording from the picture planetary to the hidden zone $[8,11,6,5]$. The pictures increased from BRATS (Multimodal brain Tumour Copy Segmentation Contest) (Figure. 1). Normal copy gained developed from IXI dataset and tumour pictures are found from BRATS.

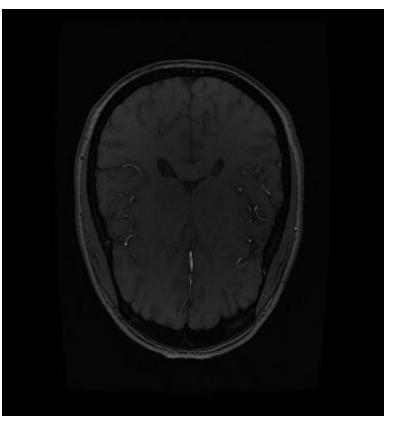

(a)

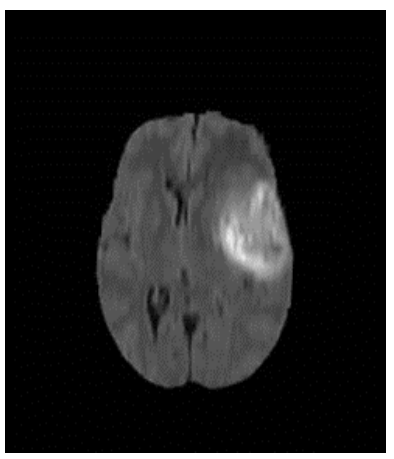

(c)

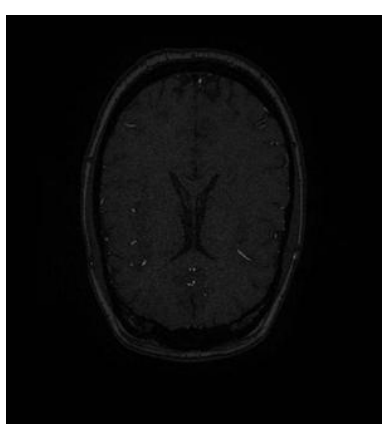

(b)

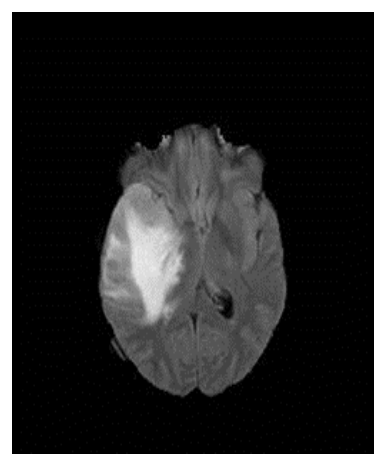

(d)
Published By:

Blue Eyes Intelligence Engineering DOI: 10.35940/ijeat.D7688.049420

Journal Website: www.ijeat.org

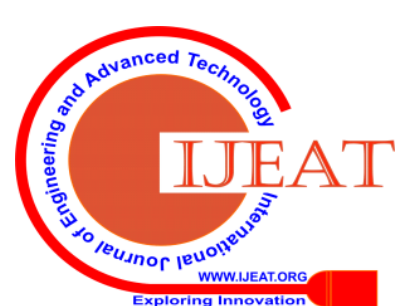




\section{Segmentation of Suspicious Region using GAN Based CNN in Brain MR Images}

Fig 1: (a) and (b)Duplicate reserved figure IXI dataset, (c) and (d) Brain Tumour Ability picture reserved from BRATS archives conventional.

Rest of the paper is set as follows, segment II comprises Generative Adversarial Networks, segment III integrate the proposed practice Gan-Based MR Picture Expansion For Brain Growth Recognition that describes experimental belongings and discussions and segment IV concludes research images with purpose directions

\section{GENERATIVE ADVERSARIAL NETWORKS}

GANs are approximately castoff for actual copy release attributable to its enormously decent authority, of producing print-pragmatic pictures. These correspondences contain duplicate decrash [11, 4], picture inpainting [4], taller-intention [18, 14], picture tint [17], manner mingling[19], signifies picture handling [12],etc. Nevertheless, recent GAN- found completely copies are typically planned for a precise scheme by specific styles [14] or damage mage roles [12], and cultured by balancing facts via attractive one picture as reach and the reverse as direction [15]. Inversely, our way can recycle the information limited in a well-instruct GAN type and also allow only GAN classical as past to altogether of the afore mentioned responsibilities without reinstruction or changes. It is value observing that our process can advantage alike or flat improved values than current GAN-primarily founded approaches which are largely qualified for a positive scheme.

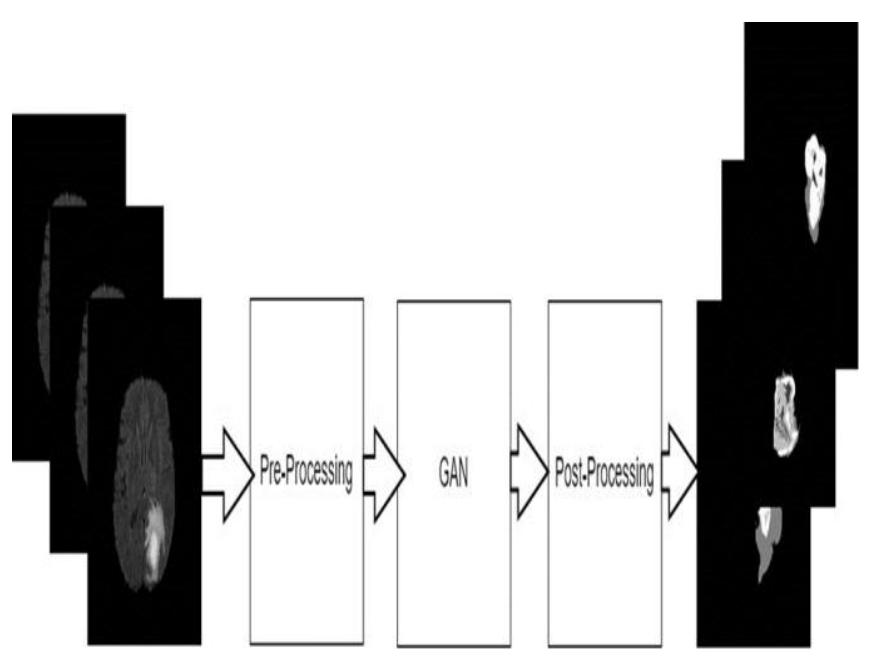

Fig 2. The overall context of the Future Technique

\section{1) Pre-processing}

Change in size, location, shape, and visible presence seriously significances GAN presentation in precise while grounding data are trivial and split, rising desired pictures needs dual-step pre-prepared:

(i) Eliminating unrelated data on health pictures (e.g., decrash/skull-deprive);

(ii) Picking and extending the residual basics to control of 2 (e.g., $256 \times 256 / 32 \times 32 \times 32$ ).

The Spatial Pyramid Pooling (SPP) sheet exclusive the separator can be custom to region off the effect of extending [1].Classical Deep Attention techniques (i.e., ordered/absorption changes) could assist GAN grounding(at the minimum flat and perpendicular reversing), composed by Webly Overseen Knowledge [2] for outward accounts.

\section{2) GAN-based Image Group}

We could mutually produce entire pictures counting uncontrolled portions (e.g., comprehensive Brain MR pics by tumours) [3] or Areas of Attention unaided (e.g., covering scratch pictures) [4] for 2 Dimension picture group, nevertheless superlative Areas of Attention unaided (e.g., 3-D lung CT nodules) [5] for 3-dimension picture group since weighty calculation control-if desired, we could glue area of attention obviously to the complete impulsive for Deep Attention. Furthermore, Advanced Rising of GANs [1] by the Wasserstein loss the usage of rise consequence [6] can produce tons of accurate tall-intent (i.e., $\geq 256 \times 256$ ) health pics. From the hidden space, whether to model features the practice of a steady or even supply does not temporarily type a large variance to Deep Attention.

\section{3) Post-processing}

GAN-effect models frequently comprise noticeable amount; nevertheless, removal such photographs by bizarre amount does not increase Deep Attention, because practicality complete by means of individuals-counting via Pictorial Turing Test [10] by means of professional surgeons [9]—isn't always powerfully related by Deep Attention act. So, use both achievement/disappointment cases for Deep Attention without further-convert, until the artificial pics surely lack virus presence.

\section{4) Image-Quality Evaluation on GAN-generated Images}

T-Divided suspect Neighbour Surrounding (t-SNE) process [2] can picture the delivery of actual and artificial pictures by way of at when embedding individuals tall-dimensional chronicles right into a 2 dimension/3-dimension extent. The Pictorial Turing Test by income of surgeons could also tentatively estimate in what way real or optimistic illness-just similar the GAN-placed artificial pictures are by means of providing, in a chance instruction, a arbitrary collection of the identical measure of definite/fake impulsive [11] each period.

\section{5) Application to Classification}

We practice GAN-performs pictures for arrangement, making even vs extreme copy (eg non-tumour vs tumour) [3] or numerous procedures of extreme pictures [10]. Frid- Adar et al. Stated improved Deep Attention act once creating labelled examples for all pathological style separately, in room of incorporating period taming to produce entirely of them after [10]. Matching among definite/artificial pictures is vital throughout clarifier grounding meanwhile addition settled-common artificial pictures details inferior presentation [3]; we are optimistic to both classically augment meekest actual or both actual/artificial pictures (empirically, the first-rate poise among physical/fake pictures is 1-to-1 or 1-to-2). Pre-refinement on ImageNet won't attain improved compassion than grounding from scrape. T-SNE could discern arrangement belongings through/deprived of Deep Attention with the aid of picturing structures removed from the outstanding coat of separately qualified clarifier.

\section{6) Application to Object Detection}

Besides, to find syndrome parts, we will deed GANs for Item Recognition; into the GANs at some point of instruction and

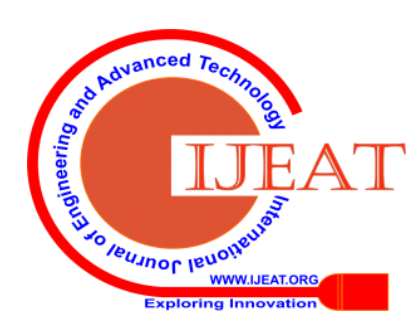


creating pictures mainly based at the explanation somewhat diverse from grounding pictures during challenging. Totaling artificial grounding pictures could attain improved understanding through extra Wrong Positives.

\section{7) Application to Segmentation}

Likewise to Entity Uncovering, we are able to protest difficult subdivision into GANs to accomplish improved Cut up add.

\section{GAN-BASED MR IMAGE AUGMENTATION FOR BRAINGROWTH DETECTION}

\section{1) Brain Growth Dataset}

As a trivial/split Deep Attention set, we combined T1c Brain axial MR pictures of four hundred Brain growth cases from numerous MRI scanners. For tumour finding, the broad dataset (four hundred patients) is separated into: (i) A grounding set (two hundred patients/two hundred impulsive); (ii) A validation set (one hundred patients/a hundred copy); (iii) A test set (a hundred patients/one hundred pics); we individual practice the tutoring common for the GAN grounding to be reasonable. We primary skull-strip all pictures through various intent, and then crop the decisive attention portions and resize them to $256 \times 256$ images. In our primary GAN-placed medicinal Deep Attention for entry finding, qualified surgeons idly explain tumours through tall-difficult and uneven bouncing filling ampules to minimalize effort.

\section{2) Proposed GAN-based Image Group}

As a initial learning, we approve an advanced grounding tactic for GANs, In the single GANs, a originator and separator are step by step rising: opening from tiny determined, novel coats variety information as instruction growths. We further condition the generator/discriminator to generate random, however practical $256 \times 256$ MR pics through tumours of arbitrary figure.

\section{3) Brain Growth Detection Using GAN based CNN}

Convolutional Neural Networks (CNNs) take the skill to achieve prominent computer-aid analysis presentation, dependent on enough marked grounding histories. Inappropriately, maximum technical imaging datasets, regularly composed after frequent scanners, are trivial and uneven. In this situation, as a Deep Attention technique, Generative Adversarial Networks (GANs) can create accurate/regular added grounding images to seal the information lack in the actual picture supply; scholars have better arrangement by way of expanding pictures with noise-to-image (e.g, arbitrary noise models to many extreme pictures) or noise-to-image GANs (e.g, a gentle picture to a malignant one). Still, no studies has stated belongings joining (i) noise-to-image GANs and picture-to-image GANs or (ii) GANs and extra profound propagative models, for in addition act improvement. After a essential search of CNN-placed tumour arrangement outcomes, also enchanting into deliberation the have an outcome on of pre-grounding on ImageNet and setting away awful-searching GAN-effect impulsive. The outcomes without a doubt presented that, combining dual-step GAN-placed Deep Attention with classic Deep Attention can radically outpace the old-style Deep Attention in tumour result alone.

\section{4) Brain Growth Detection Results}

As presented in Table 1, we've got taken 400 pics from the Deep Attention and 200 photos are taken for testing and 100 photos for Deep Attention and another 100 pics for checking out photos. Initially KNN set of rules is used to check these Deep Attention set snap shots, we've got taken $k=$ three the precision is 86.forty three and sensitivity is 81.72. The identical Deep Attention set photos and identical grounding and testing count number is taken for SVM set of rules the resultant precision is 89.ninety five and sensitivity is 85.65 . The extra GAN-generated grounding images provide more flexibility in increasing the mean Average Precision (MAP) 92.34 and sensitivity is 89.86 in go back for extra FPs according to portion; the accelerated FPs arise from also sensing blood vessels, in view that they look like the enhanced tumour sections trivial/high-acute on T1c MR image, because of the assessment manager perfest all through the blood vessels. Figure 3 shows the segmented picture after applying the proposed method .

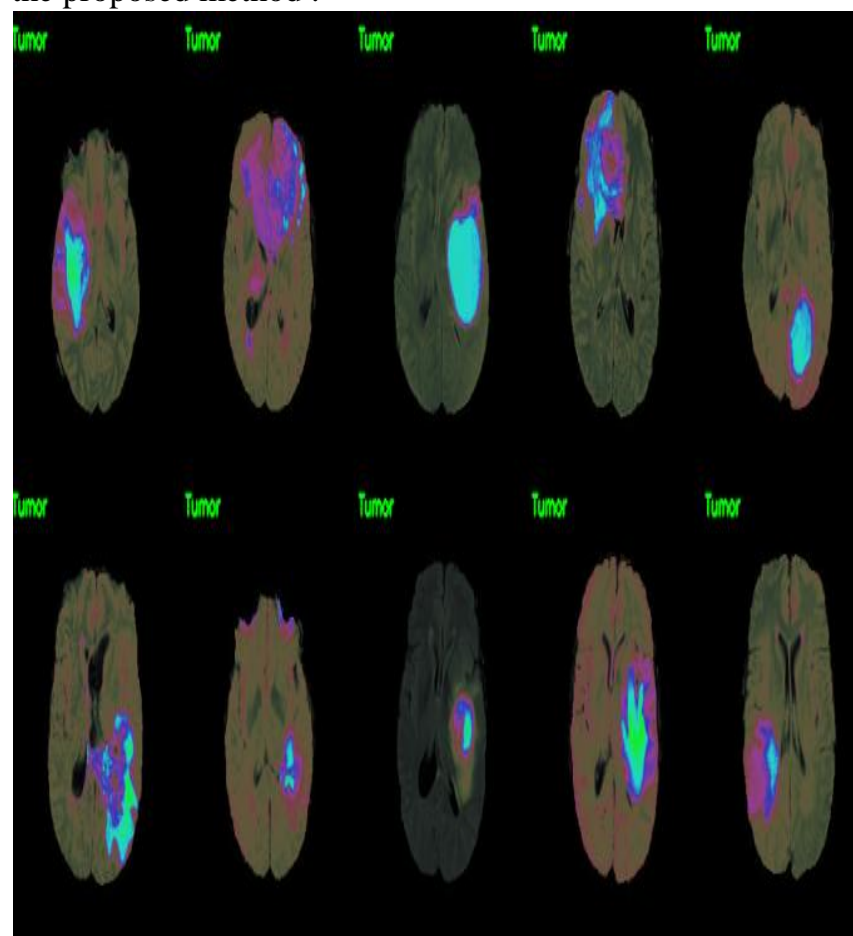

Fig 3 : Outcomes of Model breakdown on BRATS 2015 Test dataset.

Table I: Assessment of current techniques with shape-of-artwork technique 200 Images are taken for Training and Testing

\begin{tabular}{|c|c|c|}
\hline Author(s) & Precision (\%) & Sensitivity (\%) \\
\hline $\begin{array}{c}\text { KNN } \\
\text { K=3 }\end{array}$ & 86.43 & 81.72 \\
\hline SVM & 88.75 & 83.86 \\
\hline NN & 89.96 & 85.65 \\
\hline
\end{tabular}

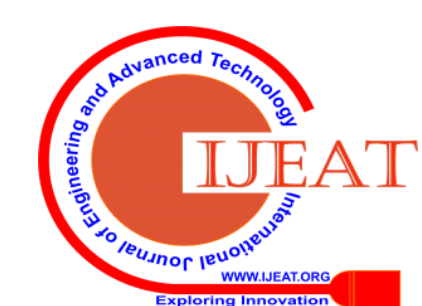




\section{Segmentation of Suspicious Region using GAN Based CNN in Brain MR Images}

\begin{tabular}{|c|c|c|}
\hline $\begin{array}{c}\text { Proposed } \\
\text { Method (GAN) }\end{array}$ & 92.34 & 89.86 \\
\hline
\end{tabular}

\section{CONCLUSION}

In conclusion, on the evidence of our practice and exposure inside the field, we verified GAN_CREATED Health Image Growth, in combination by the guiles to support cataloging/element finding/ subdivision presentation. By means of them as an sample, we likewise exhibited that our innovative GAN container create $256 \times 256$ MR pictures through mind conversion of arbitrary figure in the probability, clearly at chosen place/scope, and realize extreme concern in tumor finding-notwithstanding trivial/split grounding evidences from a pair of MRI scanners and idle explanation by means of tall-tough lively cases/probability. From the detection outcome of Brain Growth Detection Using GAN based CNN have tall Precision of 92.34(\%) and Sensitivity 89.86(\%) when as linked with extra methods. From these experiments, the proposed system has precise copy and our novel GAN-primarily founded practical Brain MR photo group technique throws slight on investigative and predictive health plans; forthcoming exploration on this correspondences are to support our inspiring outcomes.

\section{REFERENCES}

1. Andrew Brock, Jeff Donahue, and Karen Simonyan. Large scale gan grounding for tall fidelity natural image synthesis. ICLR, 2019. 1 image translation. In CVPR, 2018.

2. Antonia Creswell and Anil Anthony Bharath. Inverting the generator of a generative adversarial network. TNNLS,2018.

3. Deep Attentionvid Bau, Jun-Yan Zhu, Jonas Wulff, William Peebles, Hendrik Strobelt, Bolei Zhou, and Antonio Torralba. Inverting layers of a large generator. In ICLR Workshop, 2019.

4. Dong-Wook Kim, Jae Ryun Chung, and Seung-Won Jung. Grdn: Grouped residual dense network for real image denoising and gan-based real-world noise modeling. In CVPR Workshop, 2019

5. Fang chang Ma,UlasAyaz,and Sertac Karaman. Invertibility of convolutional generative networks from partial measurements. In NeurIPS, 2018.

6. Frid-A Deep Attentionr M, Diamant I, Klang E et al: GAN-based artificial medical image augmentation for increased CNN performance

7. Goodfellow I, Pouget-Abadie J, Mirza M et al: Generative adversarial nets. In Advances in Neural Information Processing Systems (NIPS): 2672-2680, 2014

8. Gulrajani I, Ahmed F, Arjovsky M et al: Improved grounding of Wasserstein GANs. arXiv preprint arXiv: 1704.00028, 2017

9. Guim Perarnau, Joost Van De Weijer, Bog Deep Attentionn Raducanu, and Jose M 'Alvarez. Invertible conditional gans for image editing. In NeurIPS Workshop, 2016

10. Han C, Hayashi H, Rundo R et al: GAN-based artificial BrainMR image group. In Proc. IEEE International Symposium on Biomedical Imaging (ISBI): 734-738, 2018

11. Jing wen Chen, Jiawei Chen, Hongyang Chao, and Ming Yang. Image blind denoising with generative adversarial network based noise modeling. In CVPR, 2018

12. Jin D, Xu Z, Tang Y et al: CT-realistic lung nodule simulation from 3D conditional generative adversarial networks for robust lung segmentation. In Proc. International Conference on Medical Image Computing and Computer-Assisted Intervention (MICCAI): 732-740, 2018

13. Jun-Yan Zhu, Philipp Kr"ahenb"uhl, Eli Shechtman, and Alexei A Efros. Generative visual manipulation on the natural image manifold. In ECCV, 2016.

14. Maaten L V D, Hinton G: Visualizing Deep Attentionta using t-SNE. Mach. Learn. Res. 9: 2579-2605, 2008
15. Navarro F, Conjeti S, Tombari F et al: Webly supervised learning for skin lesion classification. In Proc. International Conference on Medical Image Computing and Computer-Assisted Intervention (MICCAI): 398-406, 2018robust lung segmentation. In Proc. International Conference on Medical Image Computing and Computer-Assisted

16. Ouyang $\mathrm{X}$, Cheng $\mathrm{Y}$, Jiang $\mathrm{Y}$ et al: Pedestrian-Synthesis-GAN: Generating pedestrian Deep Attentionta in real scene and beyond. arXiv

17. Redmon J and Farhadi A: YOLOv3: An incremental improvement. arXiv preprint arXiv: 1804.02767, 2018

18. Salimans T, Goodfellow I, Zaremba W et al: Improved techniques for grounding GANs. In Advances in Neural Information

19. Zachary C Lipton and Subarna Tripathi. Precise recovery of latent vectors from generative adversarial networks. InICLR Workshop, 2017.
Published By:

Blue Eyes Intelligence Engineering

\& Sciences Publication

DOI: 10.35940/ijeat.D7688.049420

Journal Website: www.ijeat.org 